\title{
On the Possibility of Continuous, Paretian and Egalitarian Evaluation of Infinite Utility Streams*
}

\author{
Chiaki Hara
}

Institute of Economic Research, Kyoto University

\section{Tomoichi Shinotsuka}

Graduate School of Humanities and Social Sciences, University of Tsukuba

\section{Kotaro Suzumura}

Institute of Economic Research, Hitotsubashi University

and

\section{Yongsheng $\mathrm{Xu}$}

Andrew Young School of Policy Studies, Georgia State University

and

Central University of Finance and Economics

August 12, 2006

\section{Corresponding Author}

Professor Kotaro Suzumura

Institute of Economic Research

Hitotsubashi University

Naka 2-1, Kunitachi, Tokyo 186-8603, Japan

Phone \& Fax: 81-42-580-8353 E-mail: suzumura@ier.hit-u.ac.jp

\footnotetext{
${ }^{*}$ This paper is an outgrowth of our joint research which was conducted as a part of the Project on Intergenerational Equity under the auspices of the Grant-in-Aid for Scientific Research from the Ministry of Education, Culture, Sports, Science and Technology of Japan. Thanks are due to Geir Asheim, Kaushik Basu, Walter Bossert, Hajime Hori, Tomoki Inoue, Mitsunori Noguchi and Koichi Tadenuma with whom we had several discussions on this and related issues. Needless to say, no one other than ourselves should be held responsible for any defect of this paper which may still remain.
} 


\begin{abstract}
There exists a utilitarian tradition à la Sidgwick of treating equal generations equally in the form of anonymity. Diamond showed that no social evaluation ordering over infinite utility streams satisfying the Pareto principle, Sidgwick's equity principle, and the axiom of continuity exists. We introduce two versions of egalitarianism in the spirit of the Pigou-Dalton transfer principle and the Lorenz domination principle, and examine their compatibility with the weak Pareto principle in the presence of a semi-continuity axiom. The social evaluation relation is not assumed to be either complete or transitive, yet Diamond's impossibility strenuously resurfaces.
\end{abstract}

JEL Classification Nos.: D63, D71 


\section{Introduction}

There is a strong utilitarian tradition of treating otherwise equal generations equally. As Sidgwick (1907, p.414) put it, "the time at which a man exists cannot affect the value of his happiness from a universal point of view." However, a serious doubt was raised by Koopmans (1960) on the sustainability of this standpoint by showing that the rational, continuous, and stationary evaluation of infinite allocation programs cannot but exhibit a phenomenon which he christened impatience, viz., the preference for advancement along the time axis of an outcome yielding higher utility vis-à-vis another outcome yielding lower utility. This intriguing thesis was elaborated further by Diamond (1965) into a general impossibility theorem to the effect that there exists no social evaluation ordering over the set of infinite utility streams which satisfies the Pareto principle, the equity principle à la Sidgwick in the form of anonymity, and continuity with respect to the sup topology. His work was followed by, among others, Campbell (1985), Lauwers (1997), Shinotsuka (1998), and Svensson (1980), who examined the sensitivity of Diamond's theorem on the choice of underlying topology. Basu and Mitra (2003) dealt with the case where social evaluation orderings need not be continuous, but they can be represented by numerical functions. Recent study by Zame (2006) showed that there exists no social evaluation ordering satisfying the Pareto principle and equity principle in a framework where the axiom of choice is not assumed, and every set of real numbers is Lebesgue measurable.

Recollect that the equity principle à la Sidgwick does not embody any preference for egalitarian distribution of utilities among generations. Recently, there have been attempts to introduce equity principles that incorporate preferences for egalitarian distribution of utilities among generations. For example, Asheim and Tungodden (2004) and Bossert, Sprumont and Suzumura (2005) extended Hammond's (1976) equity axiom to the context of ranking infinite utility streams. In this paper, we introduce two versions of distributional egalitarianism in the spirit of Atkinson (1970) and Sen (1997), viz., the Pigou-Dalton transfer principle and the Lorenz domination principle. While the Pigou-Dalton transfer principle is concerned with the comparison between two utility streams differing from each other only in two generations, the Lorenz domination principle is concerned with the comparison between two utility streams differing from each other in any finitely many generations. We then examine their compatibility with the weak, rather than strong, Pareto principle in the presence of a semi-continuity axiom, while retaining the sup topology. The social evaluation relation is not assumed to be complete or transitive. Nevertheless, we show that the non-existence results strenuously come to the fore for both of these two principles.

Apart from this Introduction, this paper consists of five sections. Section 2 introduces our notation, model and axioms. Section 3 introduces the first class of distributional equity axioms in the spirit of the Pigou-Dalton transfer principle, and establishes impossibility theorems in the presence of upper semi-continuity with respect to the sup topology, and acyclicity of the social evaluation relation. Section 4 introduces the second class of distributional equity axioms in the spirit of the Lorenz domination principle, and establishes 
impossibility theorems in the presence of upper semi-continuity with respect to the sup topology, without imposing any rationality axiom on the social evaluation relation. In section 5, we examine the extent to which our impossibility theorems are robust with respect to the choice of alternative topologies. Section 6 concludes with some interpretative remarks.

\section{Notation, Model and Axioms}

Let $\mathbb{R}$ and $\mathbb{N}$ denote the set of all real numbers, and the set of all positive integers, respectively. Let $l^{\infty}$ be the set of all bounded infinite sequences of real numbers. In what follows, $X \subset l^{\infty}$ is the set of all infinite utility streams, viz., $\boldsymbol{x}=\left(x_{1}, x_{2}, \ldots, x_{n}, \ldots\right) \in X$ denotes an infinite sequence of utilities, where $x_{n} \in \mathbb{R}$ denotes the utility of generation $n \in \mathbb{N}$. For all $\boldsymbol{x}=\left(x_{1}, x_{2}, \ldots, x_{n}, \ldots\right)$, $\boldsymbol{y}=\left(y_{1}, y_{2}, \ldots, y_{n}, \ldots\right) \in X, \boldsymbol{x} \geq \boldsymbol{y}$ means that $x_{n} \geq y_{n}$ for all $n \in \mathbb{N} ; \boldsymbol{x}>$ $\boldsymbol{y}$ means that $\boldsymbol{x} \geq \boldsymbol{y}$ and $\boldsymbol{x} \neq \boldsymbol{y} ; \boldsymbol{x}>>\boldsymbol{y}$ means that $x_{n}>y_{n}$ for all $n \in$ $\mathbb{N}$. Denote by $l_{+}^{\infty}$ the set of all $\boldsymbol{x} \in l^{\infty}$ with $\boldsymbol{x} \geq \mathbf{0}$. We assume that $X \neq \varnothing$ and $X+l_{+}^{\infty} \subseteq X$. The sup distance being invariant under translation, we may moreover assume that $l_{+}^{\infty} \subseteq X$. The sup distance between $\boldsymbol{x}$ and $\boldsymbol{y}$ is defined by

$$
d_{s}(\boldsymbol{x}, \boldsymbol{y})=\sup _{n}\left|x_{n}-y_{n}\right|,
$$

which induces the sup topology on the space $X$.

Let $R$ be a social evaluation relation defined over $X$, viz., $\boldsymbol{x} R \boldsymbol{y}$ for any pair $\boldsymbol{x}, \boldsymbol{y} \in X$ means that the infinite utility stream $\boldsymbol{x}$ is judged to be at least as good as another infinite utility stream $\boldsymbol{y} . P(R)$ denotes the asymmetric part of $R$, viz., for all $\boldsymbol{x}, \boldsymbol{y} \in X, \boldsymbol{x} P(R) \boldsymbol{y}$ holds if and only if $\boldsymbol{x} R \boldsymbol{y}$ and not $\boldsymbol{y} R \boldsymbol{x}$. For any fixed $\boldsymbol{x} \in X$, define the lower contour set of $P(R)$ at $\boldsymbol{x} \in X$ by

$$
L_{P(R)}(\boldsymbol{x})=\{\boldsymbol{y} \in X \mid \boldsymbol{x} P(R) \boldsymbol{y}\},
$$

$R$ is said to be complete if and only if, for all $\boldsymbol{x}, \boldsymbol{y} \in X, \boldsymbol{x} R \boldsymbol{y}$ or $\boldsymbol{y} R \boldsymbol{x}$ holds. $R$ is said to be transitive if and only if, for all $\boldsymbol{x}, \boldsymbol{y}, \boldsymbol{z} \in X, \boldsymbol{x} R \boldsymbol{y}$ and $\boldsymbol{y} R \boldsymbol{z}$ imply $\boldsymbol{x} R \boldsymbol{z} . R$ is said to be an ordering if and only if it satisfies completeness as well as transitivity. Unlike most of the preceding work along the line of Koopmans and Diamond, where the social evaluation relation is assumed to be an ordering on $X$, this paper requires a much weaker property of acyclicity in Section 3, which is defined as follows. For any $t \in \mathbb{N}$, a finite subset $\left\{\boldsymbol{x}^{1}, \boldsymbol{x}^{2}, \ldots, \boldsymbol{x}^{t}\right\}$ of $X$ is called a $P(R)$-cycle of order $t$ if and only if $\boldsymbol{x}^{1} P(R) \boldsymbol{x}^{2}, \boldsymbol{x}^{2} P(R) \boldsymbol{x}^{3}, \ldots, \boldsymbol{x}^{t} P(R) \boldsymbol{x}^{1}$ hold. $R$ is said to be acyclic if and only if there exists no $P(R)$-cycle of any order $t$, where $2<t<+\infty$. It is clear that the transitivity of $R$ implies the acyclicity thereof, and the converse implication does not hold in general. Even acyclicity is not required in Section 4, while both completeness and transitivity are required in parts of Section 5 .

Concerning the continuity requirement on $R$, we will invoke the following upper semi-continuity axiom, which is weaker than Diamond's full continuity axiom.

Upper Semi-Continuity with Respect to the Sup Topology (USCs) 
For all $\boldsymbol{x} \in X, L_{P(R)}(\boldsymbol{x})$ is open with respect to the sup topology on $X$.

Another axiom, which is often invoked in the literature, is the following:

Strong Pareto Principle (SP)

For all $\boldsymbol{x}, \boldsymbol{y} \in X$, if $\boldsymbol{x}>\boldsymbol{y}$, then $\boldsymbol{x} P(R) \boldsymbol{y}$.

Although the Strong Pareto Principle has been seldom challenged in the literature in the context of economies with finite population, it has much less appeal in the context of economies with infinite population. However, the following weaker variant thereof seems to be founded on much safer ground.

Weak Pareto Principle (WP)

For all $\boldsymbol{x}, \boldsymbol{y} \in X$, if $\boldsymbol{x}>>\boldsymbol{y}$, then $\boldsymbol{x} P(R) \boldsymbol{y}$.

It should be noted that, throughout this paper, a weak preference relation $R$ is used in formulating the axioms of rationality, egalitarianism and continuity. This formulation is adopted in order to facilitate comparisons with other work, but all the axioms and the methods of proof may be given in terms of the asymmetric part thereof, viz., the strict preference relation $P$.

\section{Pigou-Dalton Transfer Principle and Its Variant}

In contrast with the equity principle à la Sidgwick and Diamond in the form of anonymity, we are now introducing an axiom which embodies a form of preference for egalitarian distribution of utilities among generations (see also Bossert, Sprumont and Suzumura (2005)). To be precise, our axiom reads as follows:

Pigou-Dalton Transfer Principle (PDT)

For any $\boldsymbol{x}, \boldsymbol{y} \in X$, and any pair of positive integers $i, j \in \mathbb{N}$, if there exists an $\epsilon>0$ such that

$$
y_{i}=x_{i}+\epsilon \leq x_{j}-\epsilon=y_{j}, x_{k}=y_{k} \text { for all } k \in \mathbb{N} \backslash\{i, j\}
$$

holds, then $\boldsymbol{y} P(R) \boldsymbol{x}$ must also hold.

Although the Pigou-Dalton Transfer Principle may be construed to be egalitarian in nature in that the utility sacrifice made by generation $j$, which is rewarded by the utility gain of the same size accruing to generation $i$, is judged to be socially beneficial, it may make even more sense to require that the intergenerational transfer should be judged socially beneficial if the gain may be larger than the sacrifice. We are thus led to the following:

Altruistic Equity Principle (AE)

For any $\boldsymbol{x}, \boldsymbol{y} \in X$, and any pair of positive integers $i, j \in \mathbb{N}$, if there exist an $\epsilon>0$ and a $\delta>0$ such that $\delta<\epsilon$ and

$$
y_{j}=x_{j}+\epsilon \leq x_{i}-\delta=y_{i}, x_{k}=y_{k} \text { for all } k \in \mathbb{N} \backslash\{i, j\}
$$


holds, then $\boldsymbol{y} P(R) \boldsymbol{x}$ must also hold.

Although PDT and AE are in general logically independent, PDT is at least as strong as $\mathbf{A E}$ if $R$ is transitive and $\mathbf{S P}$ holds. To verify this fact, let $\boldsymbol{x}, \boldsymbol{y} \in X$ and $i, j \in \mathbb{N}$ be such that there exist positive numbers $\epsilon$ and $\delta$ with $\delta<\epsilon$ satisfying $y_{j}=x_{j}+\epsilon \leq x_{i}-\delta=y_{i}$ and $x_{k}=y_{k}$ for all $k \in \mathbb{N} \backslash\{i, j\}$. Consider $\boldsymbol{y}^{*} \in X$ such that $y_{k}^{*}=y_{k}$ for all $k \in \mathbb{N} \backslash\{j\}$ and $y_{j}^{*}=y_{j}-(\epsilon-\delta)=$ $x_{j}+\delta$. It follows from PDT that $\boldsymbol{y}^{*} P(R) \boldsymbol{x}$, whereas SP implies $\boldsymbol{y} P(R) \boldsymbol{y}^{*}$. By virtue of the transitivity of $R$, we are led to $\boldsymbol{y} P(R) \boldsymbol{x}$. Thus, $\mathbf{A E}$ is implied by PDT in this case. ${ }^{1}$

Weak though PDT and AE may seem to be, we are thereby led to the following impossibility theorems.

\section{Theorem 1}

There exists no acyclic social evaluation relation $R$ satisfying PDT, WP, and USCs.

\section{Theorem 2}

There exists no acyclic social evaluation relation $R$ satisfying $\mathbf{A E}, \mathbf{W P}$, and USCs.

\section{Proof of Theorem 1 and Theorem 2}

Denote by $c_{++}^{0}$ the set of all strictly positive sequences converging to $\mathbf{0}$, which is a subset of $l^{\infty}$. Define a mapping $\varphi: c_{++}^{0} \rightarrow c_{++}^{0}$ as follows: Let $\boldsymbol{x} \in c_{++}^{0}$ and define

$$
\bar{I}=\left\{i=1,2, \ldots \mid x_{i}>\frac{2\|\boldsymbol{x}\|}{3}\right\} \text { and } \underline{I}=\left\{i=1,2, \ldots \mid x_{i}<\frac{\|\boldsymbol{x}\|}{3}\right\},
$$

where $\|\boldsymbol{x}\|=d_{s}(\boldsymbol{x}, \mathbf{0})$. Since $\underline{I}$ is non-empty, we can write $\underline{I}=\left\{k_{1}, k_{2}, \ldots\right\}$, with $k_{1}<k_{2}<\cdots$. Since $\bar{I}$ is non-empty and finite, we can write $\bar{I}=\left\{j_{1}, j_{2}, \ldots, j_{H}\right\}$, with $j_{1}<j_{2}<\cdots<j_{H}$. Then define

$$
\varphi_{i}(\boldsymbol{x})=\left\{\begin{array}{cl}
\frac{2\|\boldsymbol{x}\|}{3} & \text { if } i=j_{h} \text { for some } h \in\{1, \ldots, H\} \\
x_{i}+\left(x_{j_{h}}-\frac{2\|\boldsymbol{x}\|}{3}\right) & \text { if } i=k_{h} \text { for some } h \in\{1, \ldots, H\} \\
x_{i} & \text { otherwise }
\end{array}\right.
$$

and define $\varphi(\boldsymbol{x})=\left(\varphi_{1}(\boldsymbol{x}), \varphi_{2}(\boldsymbol{x}), \ldots\right)$. Then $\|\varphi(\boldsymbol{x})\|=2\|\boldsymbol{x}\| / 3$. Moreover, if $R$ satisfies PDT, then there is an increasingly (strictly) preferable sequence of utility streams, of length $H$, that starts with $\boldsymbol{x}$ and ends up with $\varphi(\boldsymbol{x})$.

For any $n \in \mathbb{N}$, we can apply this result $n$ times to show that $\left\|\varphi^{n}(\boldsymbol{x})\right\|=$ $(2 / 3)^{n}\|\boldsymbol{x}\|$ for every $\boldsymbol{x} \in c_{++}^{0}$ and every $n$, and hence that $\varphi^{n}(\boldsymbol{x}) \rightarrow \mathbf{0}$ as $n \rightarrow \infty$ with respect to the sup topology for every $\boldsymbol{x} \in c_{++}^{0}$. Moreover, if $R$ satisfies PDT, then there is an increasingly (strictly) preferable sequence of utility streams, of finite length, that starts with $\boldsymbol{x}$ and ends up with $\varphi^{n}(\boldsymbol{x})$.

\footnotetext{
${ }^{1}$ It is clear that this property holds true under a weaker requirement of quasi-transitivity of $R$, viz., transitivity of $P(R)$.
} 
We can now prove Theorem $\mathbf{1}$ by a contradiction argument. Take any $\boldsymbol{x} \in c_{++}^{0}$. Then, by WP, $\boldsymbol{x} P(R) \mathbf{0}$. Hence, by USCs, $\boldsymbol{x} P(R) \varphi^{n}(\boldsymbol{x})$ for every sufficiently large $n$. On the other hand, since, by PDT, there is an increasingly (strictly) preferable sequence of utility streams, of finite length, that starts with $\boldsymbol{x}$ and ends up with $\varphi^{n}(\boldsymbol{x})$, the acyclicity implies that not $\boldsymbol{x} P(R) \varphi^{n}(\boldsymbol{x})$ for any $n$. This is a contradiction.

Theorem 2 can be proved by using the same argument and adding $\|\boldsymbol{x}\| / 6$ to $x_{i}+\left(x_{j_{h}}-2\|\boldsymbol{x}\| / 3\right)$ in the definition of $\varphi_{i}(\boldsymbol{x})$.

\section{Lorenz Domination and Its Variant}

As in the case of egalitarianism on the atemporal income distributions, we may introduce an alternative formulation of egalitarian preferences over the set of infinite utility streams in terms of the concept of Lorenz domination. For each $\boldsymbol{x} \in X$ and each $n \in \mathbb{N}$, let ${ }_{1} \boldsymbol{x}_{n}$ be defined by ${ }_{1} \boldsymbol{x}_{n}=\left(x_{1}, x_{2}, \ldots, x_{n}\right)$. For any $n \in \mathbb{N}$, let us say that ${ }_{1} \boldsymbol{x}_{n}$ Lorenz dominates ${ }_{1} \boldsymbol{y}_{n}$ if and only if (i) $\sum_{t=1}^{n} x_{t}=\sum_{t=1}^{n} y_{t}$ and (ii) the Lorenz curve of ${ }_{1} \boldsymbol{x}_{n}$ lies uniformly above the Lorenz curve of ${ }_{1} \boldsymbol{y}_{n}$; similarly, let us say that ${ }_{1} \boldsymbol{x}_{n}$ Bentham-Lorenz dominates ${ }_{1} \boldsymbol{y}_{n}$ if and only if (i) $\sum_{t=1}^{n} x_{t}>\sum_{t=1}^{n} y_{t}$ and (ii) the Lorenz curve of ${ }_{1} \boldsymbol{x}_{n}$ lies uniformly above the Lorenz curve of ${ }_{1} \boldsymbol{y}_{n}$.

With the help of the above concepts, we are now ready to introduce the following Lorenz type domination principles.

Lorenz Domination Principle (LD)

For any $\boldsymbol{x}, \boldsymbol{y} \in X$, if there exists $n^{*} \in \mathbb{N}$ such that (1) ${ }_{1} \boldsymbol{x}_{n^{*}}$ Lorenz dominates ${ }_{1} \boldsymbol{y}_{n^{*}}$, and (2) $x_{n}=y_{n}$ for all $n \in \mathbb{N}$ such that $n>n^{*}$, then not $\boldsymbol{y} P(R) \boldsymbol{x}$.

Bentham-Lorenz Domination Principle (BLD)

For any $\boldsymbol{x}, \boldsymbol{y} \in X$, if there exists $n^{*} \in \mathbb{N}$ such that(1) ${ }_{1} \boldsymbol{x}_{n^{*}}$ Bentham-Lorenz dominates ${ }_{1} \boldsymbol{y}_{n^{*}}$, and (2) $x_{n}=y_{n}$ for all $n \in \mathbb{N}$ such that $n>n^{*}$, then not $\boldsymbol{y} P(R) \boldsymbol{x}$.

It may be observed that, when the transitivity of $R$ is not assumed, LD and PDT are not the same and BLD is different from AE. On the other hand, it may be noted that, if $R$ is transitive and satisfies SP, LD implies BLD even though $\mathbf{L D}$ and BLD are logically independent in general. ${ }^{2}$ The proof of this fact is similar to the proof for establishing the similar relation between PDT and AE.

We note that LD (and BLD) can be significantly weakened, without affecting the subsequent analysis, to the following condition: For any $\boldsymbol{x} \in X$ and $\boldsymbol{y} \in X$, if $\boldsymbol{x} \neq \boldsymbol{y}$ and there exists $n^{*} \in \mathbb{N}$ such that $(1) x_{n}=\left(y_{1}+y_{2}+\ldots+y_{n^{*}}\right) / n^{*}$ for every $n \leq n^{*}$ and $(2) x_{n}=y_{n}$ for every $n>n^{*}$, then not $\boldsymbol{y} P(R) \boldsymbol{x}$. That is, it suffices for $R$ to prefer the constant stream of the average utility level (for finitely many generations).

\footnotetext{
${ }^{2}$ This property holds true under the weaker rationality requirement of quasi-transitivity.
} 
As the following two results show, in the presence of semi-continuity axiom and without any rationality requirement imposed on $R$, it cannot simultaneously satisfy the weak Pareto principle and either of the domination principles introduced above.

\section{Theorem 3}

There exists no social evaluation relation $R$ satisfying WP, LD, and USCs.

\section{Theorem 4}

There exists no social evaluation relation $R$ satisfying $\mathbf{W P}, \mathbf{B L D}$, and USCs.

\section{Proof of Theorem 3 and Theorem 4}

For each $n \in \mathbb{N}$, define $\psi^{(n)}: c_{++}^{0} \rightarrow c_{++}^{0}$ by

$$
\psi^{(n)}(\boldsymbol{x})=(\underbrace{\frac{1}{n} \sum_{i=1}^{n} x_{i}, \ldots, \frac{1}{n} \sum_{i=1}^{n} x_{i}}_{n \text { times }}, x_{n+1}, x_{n+2}, \ldots) .
$$

Then, for every $\boldsymbol{x} \in c_{++}^{0}, \psi^{(n)}(\boldsymbol{x}) \rightarrow \mathbf{0}$ as $n \rightarrow \infty$ with respect to the sup topology. Indeed, let $\boldsymbol{x} \in c_{++}^{0}$ and $\epsilon>0$. Let $n^{*} \in \mathbb{N}$ be such that $x_{n} \leq \epsilon / 2$ for every $n>n^{*}$. Then, for $n>\max \left\{n^{*},(2\|\boldsymbol{x}\| / \epsilon) n^{*}\right\}$,

$$
\begin{aligned}
0<\frac{1}{n} \sum_{i=1}^{n} x_{i} & =\frac{1}{n}\left(\sum_{i=1}^{n^{*}} x_{i}+\sum_{i=n^{*}+1}^{n} x_{i}\right) \\
& \leq \frac{n^{*}}{n}\|\boldsymbol{x}\|+\frac{n-n^{*}}{n} \frac{\epsilon}{2} \\
& =\frac{\epsilon}{2}+\frac{n^{*}}{n}\left(\|\boldsymbol{x}\|-\frac{\epsilon}{2}\right) \\
& <\frac{\epsilon}{2}+\frac{1}{2\|\boldsymbol{x}\| / \epsilon}\left(\|\boldsymbol{x}\|-\frac{\epsilon}{2}\right) \\
& =\epsilon-\frac{\epsilon^{2}}{4\|\boldsymbol{x}\|}<\epsilon .
\end{aligned}
$$

Hence, by $n>n^{*},\left\|\psi^{(n)}(\boldsymbol{x})\right\|<\epsilon$. Thus $\psi^{(n)}(\boldsymbol{x}) \rightarrow \mathbf{0}$.

By WP, $\boldsymbol{x} P(R) \mathbf{0}$. Thus, by USCs, $\boldsymbol{x} P(R) \psi^{(n)}(\boldsymbol{x})$ for every sufficiently large $n$. On the other hand, ${ }_{1} \psi^{(n)}(\boldsymbol{x})_{n}$ (the first $n$ generations of $\psi^{(n)}(\boldsymbol{x})$ ) Lorenz dominates ${ }_{1} \boldsymbol{x}_{n}$ for every sufficiently large $n$. To verify this fact, note that, as shown above, $(1 / n) \sum_{i=1}^{n} x_{i} \rightarrow 0$ as $n \rightarrow \infty$. Also, by the definition of $c_{++}^{0}, x_{1}>0$. Hence $x_{1}>(1 / n) \sum_{i=1}^{n} x_{i}$ for every sufficiently large $n$. Thus, in particular, ${ }_{1} \psi^{(n)}(\boldsymbol{x})_{n} \neq{ }_{1} \boldsymbol{x}_{n}$ for every large enough $n$. Since ${ }_{1} \psi^{(n)}(\boldsymbol{x})_{n}$ is a constant sequence, this means that ${ }_{1} \psi^{(n)}(\boldsymbol{x})_{n}$ Lorenz dominates ${ }_{1} \boldsymbol{x}_{n}$. As for generations $n+1$ onwards, $\boldsymbol{x}$ and $\psi^{(n)}(\boldsymbol{x})$ share the same utility levels. Thus, it follows from LD that not $\boldsymbol{x} P(R) \psi^{(n)}(\boldsymbol{x})$ for every large enough $n$. This is a contradiction. This completes the proof of Theorem 3.

To prove Theorem 4 , use $(1 / n)\left(\sum_{i=1}^{n} x_{i}+1\right)$ in place of $(1 / n) \sum_{i=1}^{n} x_{i}$ when defining $\psi^{(n)}(\boldsymbol{x})$. 
Unlike Theorem 1 and Theorem 2 in the previous section, Theorem 3 and Theorem 4 do not require a social evaluation relation even to be acyclic, to say nothing of full transitivity. The intuition behind this sharp contrast may be given as follows. While PDT and AE are concerned with the comparison between two utility streams differing from each other only in two generations, LD and BLD are concerned with the comparison between two utility streams differing from each other in any finitely many generations. In proving all of these theorems, the crucial step was to construct an increasingly more egalitarian sequence of utility streams. While LD and BLD allow a social evaluation relation to directly compare any two streams along the sequence, PDT and AE do not. This is why we need acyclicity in proving Theorem $\mathbf{1}$ and Theorem 2.

\section{Robustness}

The impossibility theorems obtained so far commonly invoked the axiom of upper semi-continuity of social evaluation relation with respect to the sup topology. In view of the foregoing studies, e.g. Campbell (1985), Lauwers (1997), Shinotsuka (1998) and Svensson (1980), which showed that some of the impossibility theorems are not robust with respect to the choice of topology, it is important to check whether or not the validity of our impossibility theorems hinges on the choice of topology. This whole section is devoted to this problem. In the first sub-section, we show that our theorems can be generalized to social evaluation relation which is upper semi-continuous with respect to any locally solid linear topology. This fact can be established by showing that every locally solid linear topology on $l^{\infty}$ is equal to, or coarser than, the sup topology. This is reassuring to some extent, but we are still in the dark as to the case of topologies stronger than the sup topology. In the second sub-section, we assume that the social evaluation relation satisfies the axiom of completeness and that of transitivity, viz., it is an ordering, and identify a condition, called linear upper semi-continuity, under which the social evaluation ordering is upper semicontinuous with respect to the sup topology. The identified condition is, though fairly general, rather abstract. In the third sub-section, we obtain two corollaries giving simpler sufficient conditions for the upper semi-continuity with respect to the sup topology. These results do not settle our problem once and for all, but they seem to be enough to assure the relevance of our impossibility theorems.

5.1. For each $\boldsymbol{x} \in X$, let $|\boldsymbol{x}|$ be the infinite sequence of non-negative real numbers obtained from $\boldsymbol{x}$ by replacing each term $x_{n}$ with the absolute value thereof. A subset $A$ of $X$ is said to be solid if, for every $\boldsymbol{x}, \boldsymbol{y} \in l^{\infty},|\boldsymbol{x}|<|\boldsymbol{y}|$ and $\boldsymbol{y} \in A$, imply $\boldsymbol{x} \in A$. A linear topology on $l^{\infty}$ is said to be locally solid if it has a basis for $\mathbf{0}$ consisting of solid sets. The sup topology is locally solid, because the sets of the form $\left\{\boldsymbol{x} \in l^{\infty} \mid d_{s}(\boldsymbol{x}, \boldsymbol{y}) \leq \delta\right\}$ with $\delta>0$ define a fundamental system of neighborhoods of $\boldsymbol{y}$. See Aliprantis and Burkinshaw (1978) for the characterizations and basic properties of locally solid linear topologies. Note also that the locally solid linear topologies have been used in the literature on general equilibrium theory; see, for example, Mas-Colell (1986). 
The following result is mathematically trivial, but still worth keeping on record, as our subsequent analysis depends on it.

Lemma 1 Every locally solid linear topology $\mathscr{T}$ on $l^{\infty}$ is equal to, or coarser than, the sup topology.

\section{Proof of Lemma 1}

Let $\mathscr{T}$ be a locally solid linear topology on $l^{\infty}$. Since it is translationinvariant, it suffices to show that for every neighborhood $U$ of $\mathbf{0}$ with respect to $\mathscr{T}$, there exists a neighborhood $V$ of $\mathbf{0}$ with respect to the sup topology such that $V \subseteq U$. So let $U$ be a neighborhood of $\mathbf{0}$ with respect to $\mathscr{T}$. Since $\mathscr{T}$ is locally solid, there exists a solid neighborhood $W$ of $\mathbf{0}$ with respect to $\mathscr{T}$ such that $W \subseteq U$. Since the scalar multiplication $\delta \mapsto \delta \mathbf{1}$ is continuous with respect to $\mathscr{T}$, there exists a $\delta>0$ such that $\delta \mathbf{1} \in W$, where $\mathbf{1}$ is the infinite utility stream which repeats 1 indefinitely. Note that for every $\boldsymbol{x} \in l^{\infty},|\boldsymbol{x}| \leq \delta \mathbf{1}$ if and only if $d_{s}(\boldsymbol{x}, \mathbf{0}) \leq \delta$. Since $W$ is solid, this implies that $\left\{\boldsymbol{x} \in l^{\infty} \mid d_{s}(\boldsymbol{x}, \mathbf{0}) \leq \delta\right\} \subseteq W$. Since $\left\{\boldsymbol{x} \in l^{\infty} \mid d_{s}(\boldsymbol{x}, \mathbf{0}) \leq \delta\right\}$ is a neighborhood of $\mathbf{0}$ with respect to the sup topology, we can complete the proof by taking $V=\left\{\boldsymbol{x} \in l^{\infty} \mid d_{s}(\boldsymbol{x}, \mathbf{0}) \leq \delta\right\}$.

Back, then, to the evaluation of infinite utility streams. Concerning the continuity requirement of social evaluation relations, we introduce the upper semi-continuity with respect to an arbitrarily given topology $\mathscr{T}$ on $l^{\infty}$.

Upper Semi-Continuity with Respect to $\mathscr{T}\left(\mathbf{U S C}_{\mathscr{T}}\right)$

For all $\boldsymbol{x} \in X, L_{P(R)}(\boldsymbol{x})$ is an open set in $X$ with respect to the topology $\mathscr{T}$ in $X$.

By Lemma 1, if $\mathscr{T}$ is a locally solid linear topology, then $\mathbf{U S C}_{\mathscr{T}}$ implies USCs. Hence the impossibility theorems of Sections 3 and 4 lead to the following:

\section{Theorem 5}

Let $\mathscr{T}$ be a locally solid linear topology on $l^{\infty}$. Then, there exists no acyclic social evaluation relation $R$ which satisfies $\mathbf{W P}, \mathbf{U S C}_{\mathscr{T}}$ and either PDT or AE.

\section{Theorem 6}

Let $\mathscr{T}$ be a locally solid linear topology on $l^{\infty}$. Then, there exists no social evaluation relation $R$ which satisfies $\mathbf{W P}, \mathbf{U S C}_{\mathscr{T}}$, and either LD or BLD.

5.2. The next order of our business is to show that there is a good reason to believe that the class of social evaluation relations which are upper semi-continuous with respect to the sup topology is large enough in the context where we examine the compatibility, or the lack thereof, of continuity, the Pareto principle and the egalitarian principle. This point, if established, makes our impossibility theorems, which are technically confined to the sup topology and other coarser topologies, economically relevant beyond their prima facie restrictions. With this purpose in mind, let us begin with the class of social evaluation relations which satisfy completeness and transitivity, so that $R$ is an 
ordering, as well as another condition, called linear upper semi-continuity. It is admittedly less standard than completeness and transitivity, but similar conditions appeared in Herstein and Milnor (1953) for their study on the expected utility representation; it was also used much more recently by Inoue (2006) in the context of equilibrium analysis. As in the proof of Lemma 1, we denote by 1 the infinite stream which repeats 1 indefinitely.

\section{Linear Upper Semi-Continuity (LUSC)}

For all $\boldsymbol{x}, \boldsymbol{y} \in X,\left\{\alpha \in \mathbb{R} \mid \boldsymbol{y}+\alpha \mathbf{1} \in L_{P(R)}(\boldsymbol{x})\right\}$ is an open subset of $\{\alpha \in \mathbb{R} \mid \boldsymbol{y}+\alpha \mathbf{1} \in X\}$ (with respect to the Euclidean topology).

LUSC is nothing but the upper semi-continuity of $R$ when the comparison to a utility stream $\boldsymbol{x}$ is restricted to the line in the direction of $\mathbf{1}$ going through another utility stream $\boldsymbol{y}$. We shall explore some implications of this condition in the next sub-section.

We are now ready to state the following:

\section{Lemma 2}

If $X+l_{++}^{\infty} \subseteq X$ and if the social evaluation ordering $R$ satisfies $\mathbf{W P}$ and LUSC, then $R$ satisfies USCs.

\section{Proof of Lemma 2}

For each $\boldsymbol{x} \in X$, define $U_{R}(\boldsymbol{x})=\{\boldsymbol{y} \in X \mid \boldsymbol{y} R \boldsymbol{x}\}$. $R$ being complete, we obtain $X \backslash U_{R}(\boldsymbol{x})=L_{P(R)}(\boldsymbol{x})$. It thus suffices to show that $U_{R}(\boldsymbol{x})$ is a closed subset of $X$ with respect to the sup topology. So let $\boldsymbol{y} \in X$ and $\left(\boldsymbol{y}^{n}\right)_{n \in \mathbb{N}}$ be a sequence in $U_{R}(\boldsymbol{x})$ that converges to $\boldsymbol{y}$ with respect to the sup topology. It then suffices to show that $\boldsymbol{y} \in U_{R}(\boldsymbol{x})$.

By LUSC and completeness of $R$, the set $\left\{\alpha \in \mathbb{R} \mid \boldsymbol{y}+\alpha \mathbf{1} \in U_{R}(\boldsymbol{x})\right\}$, which we denote by $A$, is a closed subset of the set $\{\alpha \in \mathbb{R} \mid \boldsymbol{y}+\alpha \mathbf{1} \in X\}$, which we denote by $B$. Since $X+l_{++}^{\infty} \subseteq X, B \supseteq \mathbb{R}_{+}$. Then, for every $n \in \mathbb{N}$, $y+\left\|\boldsymbol{y}^{n}-\boldsymbol{y}\right\| \mathbf{1} \geq \boldsymbol{y}^{n}$. Hence, by WP,

$$
\left(\boldsymbol{y}+\left(\left\|\boldsymbol{y}^{n}-\boldsymbol{y}\right\|+\frac{1}{n}\right) \boldsymbol{z}\right) P(R) \boldsymbol{y}^{n}
$$

Since $\boldsymbol{y}^{n} \in U_{R}(\boldsymbol{x})$, transitivity of $R$ implies that

$$
\boldsymbol{y}+\left(\left\|\boldsymbol{y}^{n}-\boldsymbol{y}\right\|+\frac{1}{n}\right) \boldsymbol{z} \in U_{R}(\boldsymbol{x})
$$

Thus, $\left\|\boldsymbol{y}^{n}-\boldsymbol{y}\right\|+1 / n \in A$. Since $\left\|\boldsymbol{y}^{n}-\boldsymbol{y}\right\|+1 / n \rightarrow 0 \in B$ as $n \rightarrow \infty$, LUSC implies that $0 \in A$. That is, $\boldsymbol{y} \in U_{R}(\boldsymbol{x})$.

A similar proof appeared in Inoue (2006). Note that by an analogous argument in which we use

$$
\boldsymbol{y}-\left(\left\|\boldsymbol{y}^{n}-\boldsymbol{y}\right\|+\frac{1}{n}\right) \mathbf{1}
$$

we can show that $R$ is lower semi-continuous when restricted onto $\{x \in X \mid$ there exists a $\delta>0$ such that $x-\delta \mathbf{1} \in X\}$. 
The following impossibility theorem is an immediate consequence of Theorem 1 to Theorem 4 and Lemma 2.

\section{Theorem 7}

There exists no social evaluation ordering which satisfies WP, LUSC, and any one of PDT, AE, LD, and BLD.

5.3. We now explore two corollaries of Theorem 7 .

Note first that the linear operation $\alpha \mapsto \boldsymbol{y}+\alpha \mathbf{1}$ in the proof of Lemma 2 is continuous with respect to every linear topology $\mathscr{T}$ on $l^{\infty}$. Thus, if a social evaluation ordering $R$ satisfies $\mathbf{U S C}_{\mathscr{T}}$ for some linear topology $\mathscr{T}$, then it also satisfies LUSC. We thus obtain the following:

\section{Theorem 8}

Let $\mathscr{T}$ be a linear topology on $l^{\infty}$. Then, there exists no social evaluation ordering which satisfies $\mathbf{W P}, \mathbf{U S C}_{\mathscr{T}}$, and any one of PDT, AE, LD, and BLD.

For social evaluation orderings, Theorem 8 generalizes the impossibility theorems of Sections 3 and 4 to the case where the requirement of upper semicontinuity is with respect to any linear topology. It should be stressed that this generalization is not vacuous. Although the sup topology is the strongest topology among all the locally solid topologies on $l^{\infty}$, it is not the strongest even among the locally convex ones. Kolmogorov and Fomin (1971, Problem 10 of Section 17, Chapter 4) describes how to construct the strongest locally convex topology.

To extract a second implication of Theorem 7 , we consider the case in which the social evaluation ordering $R$ is represented by a concave utility function. We define $X^{*}$ as the set of all $\boldsymbol{x} \in X$ for which there exists a $\delta>0$ such that $\boldsymbol{x}-\delta \mathbf{1} \in X$. The theorem to be proved is the following:

\section{Theorem 9}

There exists no social evaluation ordering $R$ on the domain $X$ which is represented by a concave function $f: X \rightarrow \mathbb{R}$, and the restriction of which onto $X^{*}$ satisfies WP and any one of PDT, AE, LD, and BLD.

Note that no continuity condition is directly imposed on $R$. We will see in the proof that LUSC can be derived from the concavity of the utility function $f$.

To appreciate the significance of Theorem 9, recall that a Bergson-Samuelson social welfare function is a function that maps each possible profile of utility levels for finitely many individuals to a single social utility level. In the welfare analysis, it is common to assume that a Bergson-Samuelson social welfare function satisfies the Pareto Principle, so as to embody the idea of respecting efficiency, as well as concavity, so as to embody the idea of respecting egalitarianism. The function $f: X \rightarrow \mathbb{R}$ in Theorem 9 can be considered as an extension of the Bergson-Samuelson social welfare functions to the case of infinitely many individuals. We can then view Theorem 9 as claiming that once the Weak Pareto Principle and concavity are accepted for an infinite-dimensional BergsonSamuelson social welfare function, the upper semi-continuity with respect to the 
sup topology is no additional restriction on social welfare functions as long as the comparison is restricted onto the utility streams that are uniformly bounded away from the minimum levels in $X$.

\section{Proof of Theorem 9}

Let $\boldsymbol{x} \in X^{*}$ and define $A$ as the set of all $\alpha \in \mathbb{R}$ such that $\boldsymbol{x}+\alpha \mathbf{1} \in X$. Then $A$ is an interval with $\inf A<0$ and $\sup A=\infty$. Define $A^{*}=(\inf A, \infty)$, then $\boldsymbol{x}+\alpha \mathbf{1} \in X^{*}$ if and only if $\alpha \in A^{*}$. Define $\varphi: A \rightarrow \mathbb{R}$ by $\varphi(\alpha)=f(\boldsymbol{x}+\alpha \mathbf{1})$ for every $\alpha \in A$. Then $\varphi$ is concave on $A$ and thus continuous on $A^{*}$. Hence, for every $\boldsymbol{y} \in X,\left\{\alpha \in A^{*} \mid \varphi(\alpha)=f(\boldsymbol{x}+\alpha \mathbf{1})<f(\boldsymbol{y})\right\}$ is an open subset of $A^{*}$. Thus the restriction of $R$ onto $X^{*}$ satisfies LUSC. This theorem now follows from Lemma 2 and Theorem 7.

\section{Concluding Remarks}

Diamond's impossibility theorems on the evaluation of infinite utility streams, as well as most of the subsequent impossibility theorems in this arena, have the following structure in common: the strong Pareto principle cannot but conflict with the Sidgwick-Pigou-Diamond anonymity axiom in the presence of the technical axiom either in the form of continuity, or in the form of numerical representability of the social evaluation ordering. In sharp contrast, the impossibility theorems established in this paper show that the weak Pareto principle cannot but conflict with the egalitarian axiom either in the form of the Pigou-Dalton transfer principle, or in the form of the Lorenz domination principle in the presence of semi-continuity and weak rationality of social evaluation relation. In replacing the Sidgwick-Pigou-Diamond anonymity axiom by the Pigou-Dalton-Lorenz egalitarian axiom, our approach has much in common with Fleurbaey and Michel $(2001 ; 2003)$ and Sakai (2006).

Two further remarks on the Lorenz domination and related concepts may be in order. First, if a utility stream Lorenz-dominates another utility stream, the former can be arrived at from the latter by conducting Pigou-Dalton transfers from the richer to the poorer. In principle, this may take very many steps, but, in sharp contrast with PDT, $\mathbf{L D}$ requires a social evaluation relation to regard the Lorenz-dominating stream as more desirable than the Lorenz-dominated stream, irrespective of how many steps it may take to arrive at the former from the latter. Second, the Lorenz domination is essentially identical to the second order stochastic dominance (when the relevant set of finitely many generations is equipped with a uniform probability measure), just as the Suppes grading principle is essentially equivalent to the first-order stochastic dominance.

In conclusion, let us call the reader's attention to the following three characteristic features of our impossibility theorems.

(1) Recollect that most, if not all, existing impossibility theorems in this area invoked the strong Pareto principle rather than the weak Pareto principle. Since there is a conspicuous gap in appealingness between the weak Pareto principle and the strong Pareto principle in the present context, it is interesting to know that Theorem 1 to Theorem $\mathbf{4}$ hold with the weak Pareto principle and the weak rationality postulate of acyclicity or even less. 
(2) Although our analysis relied greatly on the continuity requirement on social evaluation relation, Basu and Mitra (2003) did not impose any such requirement. Instead, they assumed that social evaluation relations must be representable by numerical functions. Representability implies completeness and transitivity. Hence any impossibility theorem that does not impose completeness or transitivity is outside the scope of their impossibility theorems. This highlights the difference between their and our approaches, as our impossibility theorems use acyclicity, or even less, in place of completeness and transitivity. The same can be said of the approach by Zame (2006). As he himself exemplified, his non-measurability result would not hold for incomplete social evaluation relations.

(3) Our impossibility theorems are based on the conceptual framework of cardinal measurability and full intergenerational comparability of utilities. This is in conspicuous contrast with the framework used by Diamond (1965), where ordinal and intergenerationally non-comparable utilities are assumed. Our impossibility theorems are meant to suggest that the conflict between the Pareto principle, even in its weak version, and the egalitarian social preference persists even within the framework that permits cardinality and intergenerational full comparability. This is in sharp contrast with the social choice impossibility theorems due to Arrow (1951) and Sen (1970).

\section{References}

Aliprantis, C. D. and O. Burkinshaw (1978): Locally Solid Riesz Spaces, Pure and Applied Mathematics Series No.76, New York: Academic Press.

Arrow, K. J. (1951): Social Choice and Individual Values, New York: John Wiley \& Sons. Second Edition, 1963.

Atkinson, A. B. (1970): "On the Measurement of Inequality," Journal of Economic Theory 2, pp.244-263. Reprinted in Atkinson, A. B., Social Justice and Public Policy, Cambridge, Mass.: The MIT Press, 1983.

Asheim, G. B. and B. Tungodden (2004): "Resolving Distributional Conflicts between Generations," Economic Theory 24, pp. 221-230.

Basu, K. and T. Mitra (2003): "Aggregating Infinite Utility Streams with Intergenerational Equity: Impossibility of Being Paretian," Econometrica 71, pp.1557-1563.

Bossert, W., Y. Sprumont and K. Suzumura (2005): "Ordering Infinite Utility Streams," forthcoming in Journal of Economic Theory.

Campbell, D. E. (1985): "Impossibility Theorems and Infinite Horizon Planning," Social Choice and Welfare 2, pp.283-293.

Diamond, P. A. (1965): "The Evaluation of Infinite Utility Streams," Econometrica 33, pp.170-177. 
Fleurbaey, M. and P. Michel (2001): "Transfer Principles and Inequality Aversion, with an Application to Optimal Growth," Mathematical Social Sciences 42, pp.1-11.

Fleurbaey, M. and P. Michel (2003): "Intertemporal Equity and the Extension of the Ramsey Criterion," Journal of Mathematical Economics 39, pp.777802 .

Hammond, P. (1976): "Equity, Arrow's Conditions, and Rawls' Difference Principle," Econometrica 44, pp. 793-804.

Herstein, I. N. and J. Milnor (1953): "An Axiomatic Approach to Measurable Utility," Econometrica 21, pp.291-297.

Inoue, T. (2006): "Linearly Continuous Preferences and Equilibrium Analysis in a Finite Dimensional Commodity Space," unpublished manuscript, Institute of Economic Research, Kyoto University.

Kolmogorov, A. N. and S. V. Fomin (1971): Introductory Real Analysis, translated and edited by R. A. Silverman, New York: Dover Publications, INC.

Koopmans, T. C. (1960): "Stationary Ordinal Utility and Impatience," Econometrica 28, pp.287-309.

Lauwers, L. (1997): "Continuity and Equity with Infinite Horizons," Social Choice and Welfare 14, pp.345-356.

Mas-Colell, A. (1986): "The Price Equilibrium Existence Theorem in Topological Vector Lattices," Econometrica 54, pp.1039-1054.

Sakai, T. (2006): "Equitable Intergenerational Preferences on Restricted Domains," Social Choice and Welfare 27, pp.41-54.

Sen, A. K. (1970): Collective Choice and Social Welfare, San Francisco: HoldenDay. Republished, Amsterdam: North-Holland, 1979.

Sen, A. K. (1997): On Economic Inequality, Expanded edition with a substantial annexe by James E. Foster and Amartya Sen, Oxford: Clarendon Press.

Shinotsuka, T. (1998): "Equity, Continuity, and Myopia: A Generalization of Diamond's Impossibility Theorem," Social Choice and Welfare 15, pp.2130 .

Sidgwick, H. (1907): The Methods of Ethics, 7th edition, London: Macmillan and Co.

Svensson, L.-G. (1980): "Equity among Generations," Econometrica 48, pp.12511256.

Zame, W. R. (2006): "Can Utilitarianism be Operationalized?," manuscript, UCLA. 